\title{
SARS-CoV-2 genomics as a springboard for future disease mitigation in LMICs
}

\section{Sophie Belman, Senjuti Saha $₫$ and Mathew A. Beale $₫$}

\begin{abstract}
This Genome Watch highlights how the SARS-CoV-2 pandemic laid the groundwork for continued use of real-time genomic epidemiology for public health responses in low-and-middle-income countries.
\end{abstract}

Until recently, the use of pathogen sequencing to track emerging epidemics was mainly the domain of university research and public health laboratories in high-income countries. The SARS-CoV-2 pandemic has been pivotal in the expansion of real-time genomic epidemiology globally. Okeke and Ihekweazu ${ }^{1}$ recently discussed the historical reticence regarding implementation of molecular diagnostics in low-resource settings. For many countries, this reticence is dissipating following the expanded use of such diagnostics in the SARS-CoV-2 pandemic ${ }^{1}$. This has been similarly reflected in the implementation and repurposing of genomic sequencing pipelines in countries across the globe e $^{2-5}$. The Global Initiative on Sharing Avian Influenza Data (GISAID) database has become a repository for global sharing of SARS-CoV-2 genomes. Many low-and-middle-income countries (LMICs) have incorporated genomic sequencing into their public health response for the first time, which is reflected in GISAID submissions. In Bangladesh, MinION or Illumina sequencers that had previously been used for other diseases were repurposed to support SARS-CoV-2 sequencing ${ }^{3,4}$. This resulted in early generation of Bangladeshi SARS-CoV-2 genomes ${ }^{4}$ that contributed to investigation of the local reproductive number in Bangladesh among other descriptive epidemiological studies and reports ${ }^{4,6,7}$. Furthermore, the role played by mass population migration in SARS-CoV-2 dispersal across Bangladesh in 2020 was underscored by combining

genomes sequenced in Bangladesh with detailed human population migration data ${ }^{4}$. This demonstrated spatiotemporal overlaps between lineage expansions and holidayassociated human movement. In addition, this paper also recognized the dominance of Alpha and Beta variants in early 2021, influencing policy decisions ${ }^{3}$.

Nanopore sequencing and analysis of six Gambian genomes early in the pandemic showed phylogenetic proximity to European and Asian genomes ${ }^{5}$, supporting importation into Africa. A much larger retrospective analysis of genomes from 33 African countries also showed widespread initial introduction of SARS-CoV-2 primarily from European countries, subsequently shifting into intracontinental spread in mid-2020 (REF.'). Importantly, the major sources of importation events into other African countries were South Africa, Kenya and Nigeria, the countries with higher GDPs who submitted the majority of genomes ${ }^{2}$, which might reflect sampling biases ${ }^{2}$. Source attribution methods that assign geographical origin to ancestral nodes in a phylogenetic tree can be heavily influenced by biases in the completeness of databases. The rapid global dissemination of SARS-CoV-2 means new lineages may be first detected in countries they did not originate in. This results in sequencing-intensive and data-rich countries having an increased likelihood of being assigned as the ancestral source. We must be aware that global inferences on transmission can be influenced in a similar manner -

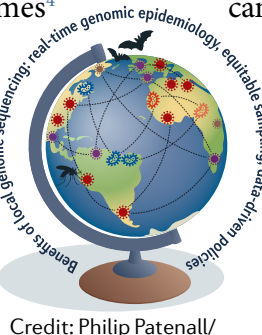

Springer Nature Limite highly sampled countries or regions are more likely to be inferred as a source of importation.

This pandemic has shown the benefits of real-time data generated through rapid sequencing and epidemiological reporting by local scientists to inform local political responses. Major public-private partnerships such as the African Pathogen Genomics Initiative and the Indian SARSCoV-2 Genomics Consortium, which support local genomic sequencing, have been developed over the past 2 years. As the pandemic wanes, interest and investment in SARS-CoV-2 and genomic sequencing for public health in LMICs may decline despite the continuing impact of disease. Maintenance of sequencing and analytical capacity will be pivotal in shifting resources post-pandemic towards diseases that represent local challenges, such as diarrhoeal disease, and endemic viruses such as dengue or chikungunya. It is only then that local genomic data can be incorporated into evidence-based policy decisions regarding treatment and intervention strategies and global research can be made truly global.

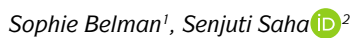
and Mathew A. Beale (iD 1 区 ${ }^{'}$ Wellcome Sanger Institute, Wellcome Genome Campus, Hinxton, UK. ${ }^{2}$ Child Health Research Foundation, Dhaka, Bangladesh.

凶e-mail: mathew.beale@sanger.ac.uk https://doi.org/10.1038/s41579-021-00664-y

1. Okeke, I. N. \& Ihekweazu, C. The importance of molecular diagnostics for infectious diseases in lowresource settings. Nat. Rev. Microbiol. 19, 547-548 (2021).

2. Wilkinson, E. et al. A year of genomic surveillance reveals how the SARS-CoV-2 pandemic unfolded in Africa. Science 374, 423-431 (2021).

3. Cowley, L. A. et al. Genomics, social media and mobile phone data enable mapping of SARS-CoV-2 lineages to inform health policy in Bangladesh. Nat. Microbiol. 6, 1271-1278 (2021).

4. Saha, S. et al. Complete genome sequence of a novel coronavirus (SARS-CoV-2) isolate from Bangladesh. Microbiol. Resour. Announc. 9, e00568-20 (2020)

5. Kanteh, A. et al. Origin of imported SARS-CoV-2 strains in The Gambia identified from whole genome sequences. PLOS ONE 16, e0241942 (2021).

6. Islam, A. et al. Assessment of basic reproduction number $\left(R_{0}\right)$, spatial and temporal epidemiological determinants, and genetic characterization of SARS-CoV-2 in Bangladesh. Infect. Genet. Evol. 92, 104884 (2021).

7. Hossain, M. E. et al. Genome sequence of a SARS-CoV-2 strain from Bangladesh that is nearly identical to United Kingdom SARS-CoV-2 variant B. 1.1.7. Microbiol. Resour. Announc. 10, e00100-21 (2021). 